\title{
Characterizing Graphite Nodule in Ductile Iron using TKD and TEM
}

\author{
Jingjing Qing, Mingzhi Xu
}

Missouri University of Science \& Technology, Materials Science \& Engineering Department, Rolla, MO, USA

Graphite is a constituent phase in the cast irons. Graphite in the ductile iron takes spheroidal/nodular morphology, because of the addition of nodulizing elements such as magnesium, cerium or rare earth elements [1-2]. The spheroidal graphite in ductile iron nucleates on heterogeneous nucleation sites [3-4]. Growth of graphite in the hypereutectic ductile iron first occurs in liquid. Solid austenite shell isolates the graphite nodule from liquid during later growth stage of the graphite. Graphite nodule from a hypereutectic ductile iron (graphite as primary phase) was cross-sectioned using Focused Ion Beam (FIB). The complex compound was exposed at the center of the graphite nodule as its heterogeneous nucleation sites. Thin foil specimen containing graphite and its nucleus was prepared from the graphite nodule using FIB. Components/phases in the complex compound were analyzed using EDX (Energy Dispersive X-ray), TKD (Transmission Kikuchi Diffraction), and TEM (Transmission Electron Microscopy). Internal structure of the graphite nodule was analyzed using TEM, which shows the stages of graphite growth in ductile irons.

The TEM bright images show that this nucleus is composed of a core and three components on the sides of the core, as shown in Figure 1. TEM selected area diffraction patterns (SADPs) were collected from each individual phase in the nucleus of the graphite nodule. Crystal structures of the phases are identified based on the SADPs, and they are compared to the TKD results. EDX analyses of the nucleus indicates that the complex compound is made of the $\mathrm{Mg}$-O core and the $\mathrm{Ca}-\mathrm{S}$ side-component and the $\mathrm{Mg}$-Al-Si-O side-components. Enrichment of nodulizing element, magnesium, is observed in the nucleus, especially in the $\mathrm{Mg}-\mathrm{O}$ core. Calcium, aluminum, silicon are additive elements in the inoculant (ferrosilicon based alloy) for ductile iron, which was introduced in the liquid metal during inoculation process. Higher concentrations of these elements that are beneficial to graphite inoculation are observed in the nucleus.

TKD is used in combination with TEM diffraction to confirm the phases in the nucleus. TKD of the nucleus is showing that the core of the nucleus ( $\mathrm{Mg}-\mathrm{O}$ compound) is faced centered cubic structure. The $\mathrm{Mg}-\mathrm{Al}-\mathrm{Si}-\mathrm{O}$ compound has an orthorhombic structure. Each of the compound is a single grain. The two $\mathrm{Mg}-\mathrm{Al}-\mathrm{Si}-\mathrm{O}$ compounds found in this sample are in twin orientation relationship. Ca-S compound degraded to amorphous phase due to the oxidation during sample handling, and thus it cannot produce usable kikuchi pattern for crystal structure indexing.

The high resolution images of the graphite and silicate interface indicates that this heterogeneous nucleus form semi-coherent interfaces with the graphite (see Figure 2(a)). The Mg-O core has a highly ordered structure, as shown in Figure 2(b). Graphite lattice grows semi-coherently on the Mg-Al-Si-O compound. Near the center of the nodule or next to the nucleus of a nodule, the graphite lattice shows high curvature, without distinct sub-boundary or sub-structure. Graphite nodules form in liquid initially during its early growth stage, independently from austenite dendrite. Austenite dendrite engulfs the graphite nodules once in contact during eutectic reaction. For a graphite nodule formed during early growth stage, graphite basal planes show continuous curvature and the growth proceeds in prismatic directions along the circumference of the graphite nodules. Later growth of the graphite nodule follows a different growth mechanism [6]. 
The later growth starts when the graphite nodule is engulfed by austenite shell, which occurs via carbon diffusion through the solid austenite shell. Local growths of graphite during later growth stage produce substructures in the graphite nodule. The substructures are separated by tilt/twin boundaries along the radial directions, and c-axis rotation faults perpendicular to the radial direction of the nodule.

References:

[1] G. M. Goodrich et al, Cast irons, Castings, in ASM Handbook, Vol. 15. ASM International, 2008.

[2] Ductile Iron Society, Ductile Iron Data, https://www.ductile.org/ductile-iron-data-2/, 2018.

[3] T. Skaland, Proceedings of the AGS Cast Iron Inoculation Conference, 2005.

[4] H. Nakae and Y. Igarashi, Materials Transactions 43 (2002), p. 2826.

[5] J. Qing, V. L. Richards, D. C. Van Aken, Carbon 116 (2017), p. 456.

[6] J. Qing, V.L. Richards, D. C. Van Aken, Transactions of the American Foundry Society 125 (2017), p. 161 .

[7] J. Qing, V. L. Richards, D. C. Van Aken, Metall. and Mat. Trans. A 47A (2016), p. 6197.

[8] J. Qing, V. L. Richards, D. C. Van Aken, Transactions of the American Foundry Society 123 (2015), p. 271.

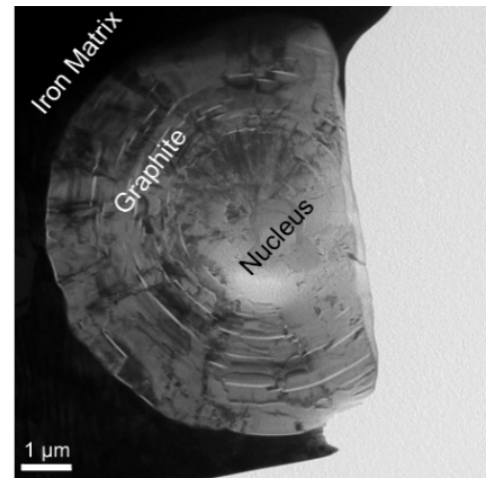

(a)

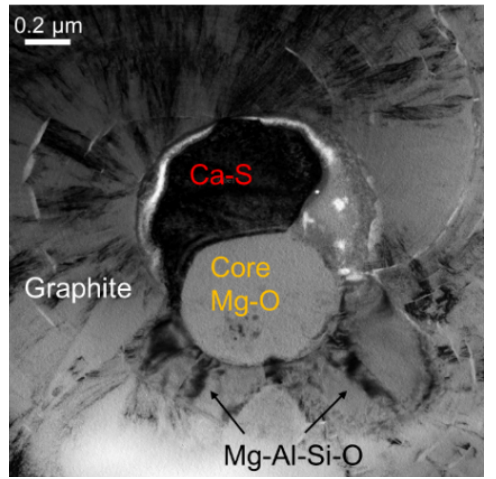

(b)

Figure 1. TEM bright field images of (a) graphite nodule in ductile iron containing heterogeneous nucleus and (b) the phases in the graphite nodule nucleus.

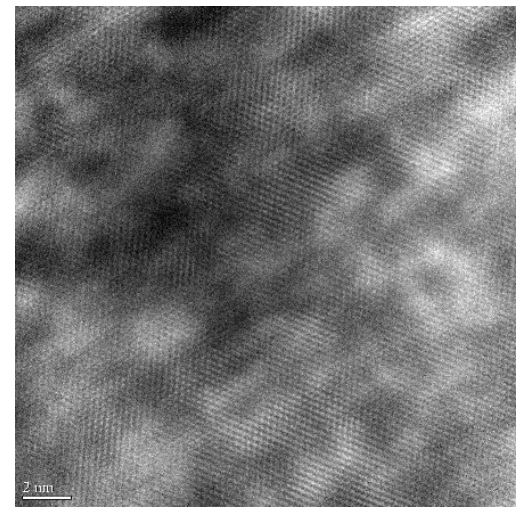

(a)

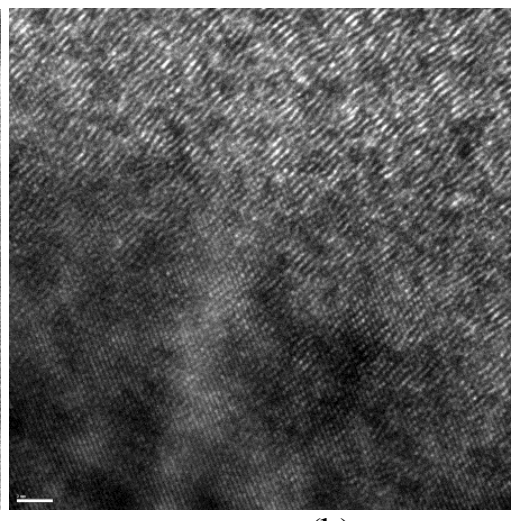

(b)

Figure 2. Lattice fringes of (a) $\mathrm{Mg}-\mathrm{O}$ core and (b) the interface between graphite (upper) and $\mathrm{Mg}-\mathrm{Al}-\mathrm{Si}$ O compound. 